\title{
LIGHT ATTENTUATION AND VISIBILITY IN BLOWING SNOW
}

by

\author{
Masao TAKEUCHI AND YoshIFUMI FuKUZawa
}

Civil Engineering Research Institute, Hokkaido Development Bureau, Sapporo, Japan 062

\section{ABSTRACT}

Visibility in blowing snow was investigated by measurements of visibility using optical targets, light attenuation of parallel beam and snow concentration and mass flux of airborne snow particles.

Visibility is thought to be inversely proportional to extinction coefficient of light according to Koschmeider's equation of visibility. However, visually observed values are not in accordance with those calculated from extinction coefficient of light when visibility is at a very low range. The extinction coefficient of light is proportional to the total cross section of airborne snow particles in a light path. The total cross section is also in proportion to concentration of airborne snow particles. There are many reported investigations of relation between visibility and snow concentration with widely differing results. The extinction coefficient of light varies with the shape and size of the particles, but cannot totally account for observed differences.

Transforming the snow concentration into mass flux of snow particles, a better relation between visibility and mass flux was found. These facts are explained in terms of the size of snow particles which are large enough to be seen Visibility through visible particles is determined not only by the homogeneous attenuation of light discovered by Koschmeider, but also by the influence of visible particles themselves and their afterimage.

\section{INTRODUCTION}

Light attenuation by snow particles has been investigated to find improved methods of measurement of snow fall intensity, or to provide continuous automatic observation of visibility in falling or blowing snow by using transmissometers. However, attenuation of light is proportional to the total cross section of snow particles or snow concentration in a light path, and simultaneous measurements of snow particles and light attenuation are rare, especially in blowing snow. Mellor (1966), Saito (1971) and Budd and others (1966) have studied visibility in falling or blowing snow in relation to snow concentration.

Visibility is inversely proportional to snow concentration; however, the results considerably disagree. The disagreement suggests that large visible particles affect visibility in falling or blowing snow.

\section{METHODS OF MEASUREMENT}

Attenuation measurements were made with a transmissometer which was set at right angles to the prevailing wind. The $1 \mathrm{KHz}$ of modulated nearly-parallel beam traversed a path $3 \mathrm{~m}$ or $74.2 \mathrm{~m}$ long, $1.5 \mathrm{~m}$ above the snow surface. Drift flux at $1.5 \mathrm{~m}$ level was measured with porous fabric bags that filtered particles from the wind stream. Visibility was observed visually by using seven black targets of a size $0.5^{\circ}$ of the visual angle from the observation point.

\section{THEORETICAL CONSIDERATION}

From Mie theory the extinction coefficient, $\sigma$, for monodispersed particles is given by

$$
\sigma=\mathrm{NKa}
$$

where $\mathrm{N}$ is the number of particles and a the geometrical cross section of the particles. The scattering area ratio of the particle, $\mathrm{K}$, is defined as the ratio of extinction cross section to the geometric cross section of the particle. As particle size increases, the value of $K$ tends toward a constant value at 2 . The value of 2 has been taken as the scattering area ratio of the snow particles (O'Brien 1970; Warner and Gunn 1969). However, snow particles are very large compared with the wave length of light so that Mie theory is not valid.

For a large value of parameter

$$
\alpha=\frac{2 \pi \mathrm{r}}{\lambda},
$$

(Brillouin 1940 suggests 160 for a typical case) where $r$ is the particle radius and $\lambda$ is the wavelength, the scattering area ratio must approach the value unity required by considerations of geometric optics.

In the region of radius of particles larger than 20 $\mu \mathrm{m}$, geometrical optics should apply for the wave length of visible light. Part of the light passes through the snow crystals so the scattering area ratio should be smaller than the value of 1 . The values of 0.352 (dry dendriti) and 0.166 (powder and columns) were measured as the scattering area ratio by O'Brien (1970). Values smaller than 1 would mean that the light attenuated by the reflection from snow particles partly passes through them.

\section{LIGHT ATTENUATION IN BLOWING SNOW}

It is possible to measure the instant value of light attenuation continuously. But it is not easy to make direct measurements of instant concentration of snow particles and their distribution. Average snow concentration was calculated from simultaneous measurements of snow flux and wind speed. The concentration of particles $\mathrm{N}$ in Equation 1 can be expressed as a function of mass concentration D at least for simple particle shapes:

and

$$
N=\frac{A_{1} K}{e^{3}} \frac{D}{y}
$$

$$
\sigma=\frac{A_{2} K}{l} \quad \frac{D}{y} \quad K<1
$$

where $\mathcal{L}$ is a characteristic linear dimension of the particle, $y$ is density of ice and $A_{1}, A_{2}$ are dimensionless constants, for a given size and shape.

The attentuation of light in traversing a distance $\mathrm{L}$ is given by the Bouguer-Lambert law in the form:

$$
B=B_{0} e^{-\sigma L}
$$

Where $B$ is the intensity at $L, B_{0}$ is the initial intensity. From Equation 4, the extinction coefficient is

$$
\sigma=\frac{1}{\mathrm{~L}} \ln \frac{1}{\mathrm{~T}}
$$

where $T\left[=\frac{B}{B_{0}}\right]$ is the transmissivity.

Observed relation between extinction coefficient and mass concentration in blowing snow is shown in Figure 1 


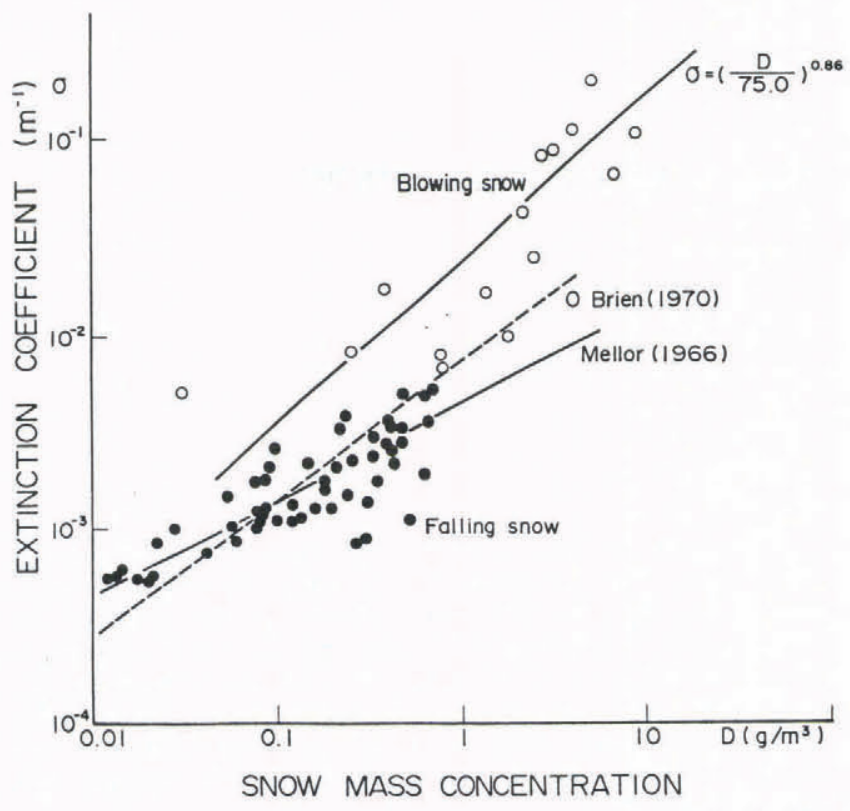

Fig.1. Observed extinction coefficient vs snow mass concentration in blowing and falling snow.

comparing with the results in falling snow (Mellor 1966; O’Brien 1970).

The extinction coeffficient in blowing snow is larger than that of falling snow at the same mass concentration because broken and rounded particles of blowing snow are smaller than falling snow particles.

\section{VISIBILITY IN BLOWING SNOW}

The visual range (visibility) $\mathbf{V}$ is presented in terms of the extinction coefficient $\sigma$ by Koschmeider on the assumption of the uniformity of both light and atmosphere, ie

$$
\mathrm{V}=\frac{1}{\sigma} \ln \frac{1}{\epsilon}
$$

where $\epsilon$ is liminal contrast (Middleton 1952).

The value of $\sigma$ is proportional to mass concentration (Equation 3) in case of monodispersed particles. The observation of visibility and mass concentration at eye level were made simultaneously in blowing snow.

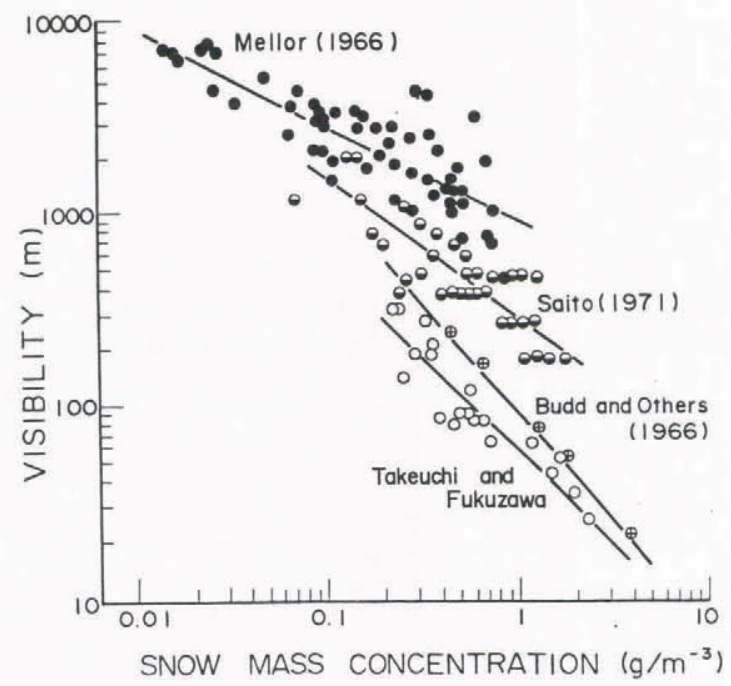

Fig.2. Visibility vs snow mass concentration. The data of Mellor (1966), Saito (1971) and Budd and others (1966) were obtained respectively in conditions of windless falling snow, falling snow in seasonal wind, and Antarctic blizzard.
Visibility related to snow concentration is compared with other visibility data in Figure 2 . Visibility is in good relation to mass concentration in all weather conditions; however, there is considerable disagreement among the results. Comparing visibility in falling snow and in blowing snow, the former is $1000 \mathrm{~m}$ and the latter is $60 \mathrm{~m}$ at the same snow concentration $\left(\mathrm{g} / \mathrm{m}^{3}\right)$. Visibility was observed visually and related to extinction coefficient measured by transmissometer in Figure 3.

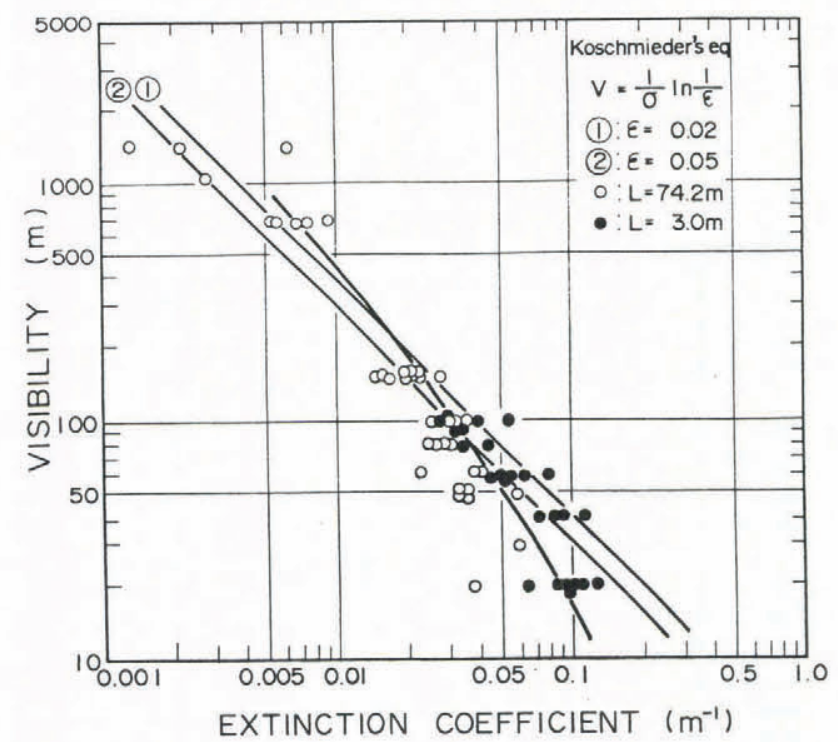

Fig.3. Visibility vs extinction coefficient.

The relation between visibility and extinction coefficient is not in accordance with Koschmieder's equation in the range of visibility lower than $150 \mathrm{~m}$. These results suggest that snow particles are very large compared with the particles which Koschmieder assumed. Snow particles are visible in the location close to an observer and the influence on the visibility by a snow particle probably differs with location of particle. A visible moving snow particle is seen like a line; Saito (1971) suggests theoretically that the faster white particles move against the black body, the stronger it stimulates the eye.

That is to say, that as the velocity of snow particles increases, the visibility observed by using black targets decreases at the same mass concentration. The mass flux of snow particles is a function of particle velocity and mass concentration so the effect of particle velocity can be expressed by mass flux though indirect. The visibility related to mass flux is shown in Figure 4. The mass flux, $\mathrm{Mf}$, at eye level was calculated by mass concentration and particle velocity as:

$$
M_{f}=D_{v} V^{2}+V_{t}^{2}
$$

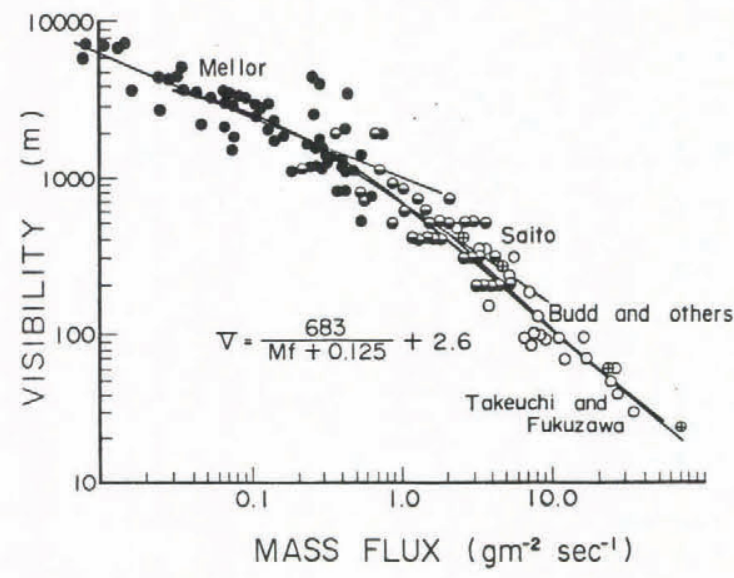

Fig.4. Visibility vs mass flux. 


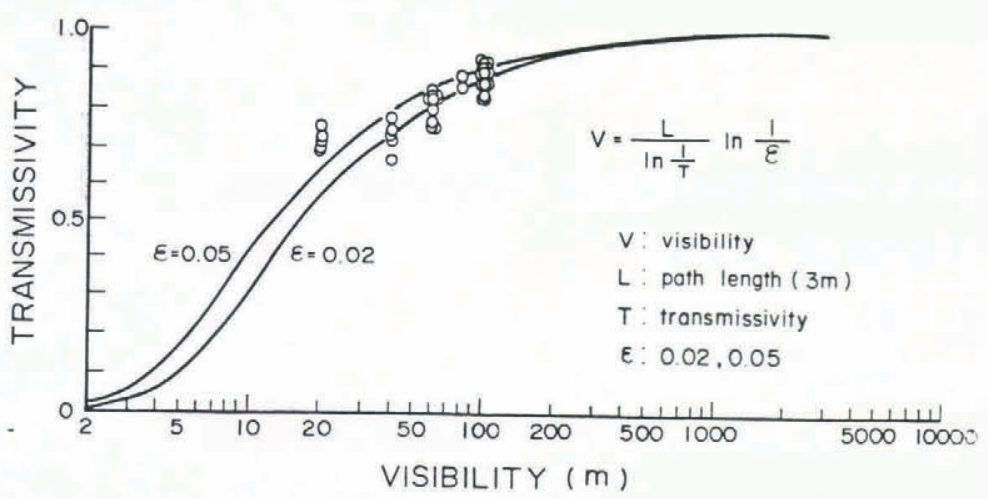

Fig.5. Visibility vs transmissivity.

where $\mathrm{V}$ is wind speed and $\mathrm{Vt}$ is the terminal velocity of snow particles. The gradient of the regression curve increases with decreasing visibility in Figure 4 , for the ratio of the range where snow particles are visible to the visibility itself increases and the influence of visible snow particles and afterimage of them is enlarged in the lower range of visibility.

\section{VISIBILITY MEASUREMENT BY TRANSMISSOMETER}

Poor visibility on road in blowing snow is the major snow problem in Hokkaido. It is very important for traffic operation to monitor visiblity. The required accuracy of visibility is not so severe as that of precipitation for practical use. A transmissometer can be used as a visibility monitor. Relation between visibility and transmissivity is derived from Koschmieder's equation and Bouguer-Lambert law as:

$$
V=\frac{L}{\ln \left(\frac{1}{T}\right)} \ln \frac{1}{\epsilon}
$$

The result of transmissivity measured by transmissometer having path length $3 \mathrm{~m}$ and visual observation of visibility is shown in Figure 5. The visibility data shift from the theoretical curve to lower visibility in the very low range. Traffic flow cannot be maintained in visibility lower than $30 \mathrm{~m}$, so the deviation is not important for practical use.

\section{CONCLUSIONS}

Geometrical optics should apply to the light attenuation by snow particles. The visible size of snow particles and their afterimage influence the visibility toward a decrease. Transmissometers are useful as visual range monitors in blowing snow.

\section{REFERENCES}

Brillouin L 1949 The scattering cross section of spheres for electromagnetic waves. Journal of Applied Physics 20(11): 1110-1125

Budd W F, Dingle W R, Radok U 1966 The Byrd snow drift project: outline and basic results. American Geophysical Union, Antarctic Research Series 9: 71-134

O'Brien M P 1970 Visibility and light attenuation in falling snow. Journal of Applied Meteorology 9(8): 671-683

Mellor M 1966 Light scattering and particle aggregation in snowstorms. Journal of Glaciology 6(44): 237-248

Middleton W E K 1952 Vision through the atmosphere. Toronto, University of Toronto Press, 250p

Saito H 1971 [Intensity of falling snow and visibility]. Report of the National Research Center for Disaster Prevention 5: 33-40 (in Japanese) 